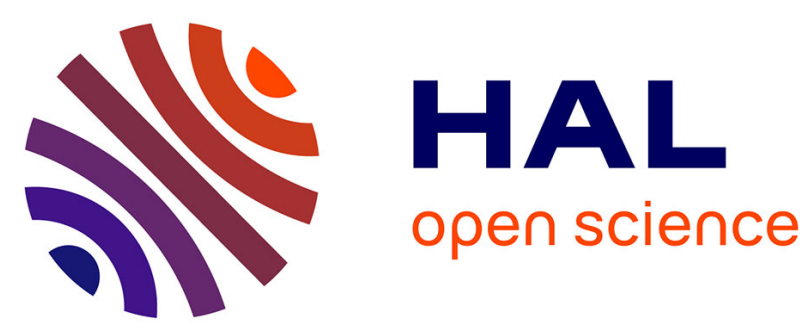

\title{
Epsilon-Near-Zero Mode for Active Optoelectronic Devices
}

Simon Vassant, Alexandre Archambault, François Marquier, Fabrice Pardo, Ulf Gennser, Antonella Cavanna, Jean-Luc Pelouard, Jean-Jacques Greffet

\section{- To cite this version:}

Simon Vassant, Alexandre Archambault, François Marquier, Fabrice Pardo, Ulf Gennser, et al.. Epsilon-Near-Zero Mode for Active Optoelectronic Devices. Physical Review Letters, 2012, 109 (23), pp.237401. 10.1103/PhysRevLett.109.237401 . hal-00785260

\section{HAL Id: hal-00785260 \\ https://hal-iogs.archives-ouvertes.fr/hal-00785260}

Submitted on 12 Apr 2013

HAL is a multi-disciplinary open access archive for the deposit and dissemination of scientific research documents, whether they are published or not. The documents may come from teaching and research institutions in France or abroad, or from public or private research centers.
L'archive ouverte pluridisciplinaire HAL, est destinée au dépôt et à la diffusion de documents scientifiques de niveau recherche, publiés ou non, émanant des établissements d'enseignement et de recherche français ou étrangers, des laboratoires publics ou privés. 


\title{
Epsilon Near Zero mode for active optoelectronic devices
}

\author{
S. Vassant, ${ }^{1,2}$ A. Archambault, ${ }^{1}$ F. Marquier, ${ }^{1}$ F. Pardo,${ }^{2}$ U. Gennser, ${ }^{2}$ A. Cavanna, ${ }^{2}$ JL. Pelouard, ${ }^{2}$ and JJ. Greffet ${ }^{1}$ \\ ${ }^{1}$ Laboratoire Charles Fabry, Institut d'Optique, CNRS, Univ. Paris-Sud, \\ 2 avenue Augustin Fresnel, 91127 Palaiseau cedex, France \\ ${ }^{2}$ Laboratoire de Photonique et Nanostructures (LPN-CNRS), Route de Nozay, F-91460 Marcoussis, France
}

\begin{abstract}
The electromagnetic modes of a GaAs quantum well between two AlGaAs barriers are studied. At the longitudinal optical phonon frequency, the system supports a phonon polariton mode confined in the thickness of the quantum well that is called Epsilon Near Zero (ENZ) mode. This ENZ mode can be resonantly excited through a grating resulting in a very large absorption localized in the single quantum well. We show that the reflectivity can be modulated by applying a voltage. This paves the way to a new class of active optoelectronic devices working in the mid and far infrared at ambiant temperature.
\end{abstract}

PACS numbers: 73.20.Mf, 78.68.+m, 42.79.Ta, 85.60.-q, 78.20.Ci

A cornerstone of nanophotonics is the enhancement of electron-photon interaction by combining field confinement and resonances as in microcavities or nanoantennas. This enhancement can also be obtained by using surface waves, e.g. surface plasmon or surface phonon polaritons $(\mathrm{SPhP})[1,2]$. Examples of applications include the enhancement of Raman scattering [3], the enhancement of fluorescence $[4,5]$, the enhancement of the radiative decay rate [6-8], localized heating [9]. More recently, a further step was done by introducing active plasmonic devices [10-13]. In the IR, SPhP allows designing resonant absorbers as demonstrated with polar semiconductors in the infrared $[14,15]$ and $\mathrm{THz}[16]$ ranges. However, it is more difficult to use surface waves to enhance interaction with electrons in a quantum well (QW), as their spatial extension is generally more than one order of magnitude larger than a typical QW thickness and therefore the electron-photon spatial overlap is far from optimum. Here, we overcome this issue by the Epsilon Near Zero (ENZ) effect, which has been discussed in a series of recent papers [17-20]. Here, we introduce an ENZ SPhP mode (hereafter called ENZ mode), which allows enhancing the incident intensity by more than two orders of magnitude in a single QW. We first discuss the ENZ mode properties in the case of a single AlGaAs/GaAs/AlGaAs QW. We then demonstrate experimentally a resonant absorption due to the ENZ mode excitation. We finally report an electrical control of this absorption that allows modulating the reflectivity at ambient temperature.

Let us first introduce the ENZ mode. If one considers an interface between two media (1 and 2), the electric displacement $\epsilon_{z z} E_{z}$ normal to the interfaces is continuous so that $E_{z 2} / E_{z 1}=\epsilon_{z z 1} / \epsilon_{z z 2}$, where $\epsilon_{i j}$ is the diagonal complex dielectric tensor and $\mathrm{E}$ the electric field. Thus if $\left|\epsilon_{z z 2}\right| \ll\left|\epsilon_{z z 1}\right|$, the field in medium 2 is enhanced compared to the field in medium 1 . We can define an enhancement factor $K_{E N Z}=\left|E_{z 2} / E_{z 1}\right|^{2}=\left|\epsilon_{z z 1} / \epsilon_{z z 2}\right|^{2}$. We now consider the $\mathrm{GaAs} / \mathrm{Al}_{0.33} \mathrm{Ga}_{0.67} \mathrm{As}$ system and show that it naturally exhibits ENZ enhancement. Our goal is to combine this effect with the resonant excitation of a $\mathrm{SPhP}$ to achieve a resonant absorption. In Fig. 1a, we plot the real part of the dielectric function of GaAs. The green (dark gray) shaded area corresponds to $K_{E N Z} \geq 50$, whereas the gray shaded area corresponds to the frequency range where GaAs and AlGaAs have the real part of their dielectric constants with opposite signs so that a SPhP can exist at a GaAs/AlGaAs interface. The ENZ and SPhP frequency range do not overlap for this geometry. However, if we consider a slab of GaAs embedded in AlGaAs (Fig. 1c), SPhP propagating at both interfaces can couple leading to a splitting of the $\mathrm{SPhP}$ dispersion relation in two branches[21]. As can be seen in Fig. 1b, the splitting increases when the slab thickness (d) decreases.

For small thicknesses (here 22nm), the frequency of the higher energy branch enters the ENZ frequency range but remains smaller than the longitudinal optical phonon frequency of $\mathrm{GaAs}\left(\omega_{\mathrm{GaAs}, \mathrm{LO}} \approx 291.55 \mathrm{~cm}^{-1}[21]\right)$, so that the condition $\operatorname{Re}\left(\epsilon_{G a A s}(\omega)\right)<0$ is fulfilled. We refer to this mode as ENZ mode. Its field distribution is depicted in Fig. 1c, for a GaAs thickness of $22 \mathrm{~nm}$ and a $7000 \mathrm{~cm}^{-1}$ wavevector. Two important features are seen: i) the field is confined in the QW; ii) the amplitude in GaAs is more than one order of magnitude larger than in $\mathrm{Al}_{0.33} \mathrm{Ga}_{0.67} \mathrm{As}$. The system bears some resemblance with two surface plasmons propagating along a thin metallic film. In both cases, we have a metal-like medium embedded in a dielectric. The ENZ-mode has the same field symmetry as the long range surface plasmon (symmetric normal electric field) [22, 23]. Despite these similarities, the ENZ mode and the long range surface plasmon have significant differences: i) a different field distribution: $|E|$ is maximum in the film for the ENZ mode whereas it is minimum in the case of the long-range surface plasmon, ii) the ENZ mode dispersion relation is almost flat for a large range of wavevectors, with $\omega \approx \omega_{\mathrm{GaAs}, \mathrm{LO}}$.

We now show how the ENZ mode can be used for optoelectronic devices. Here, we limit the discussion to the 
(a)

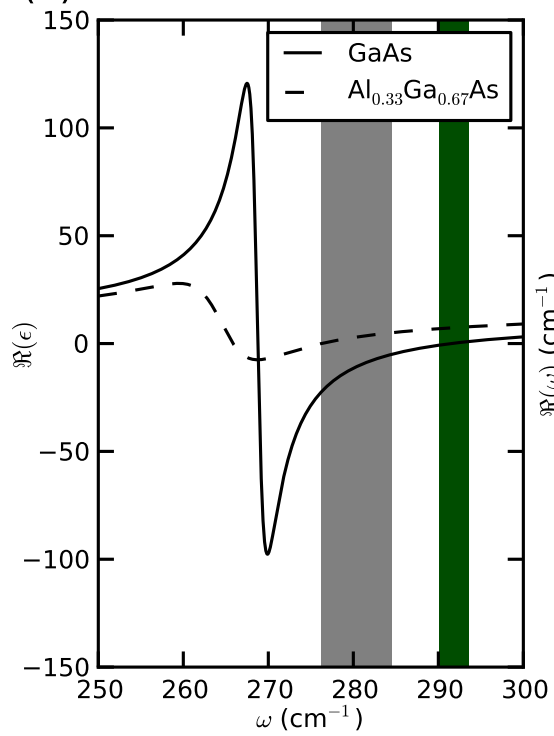

(b)

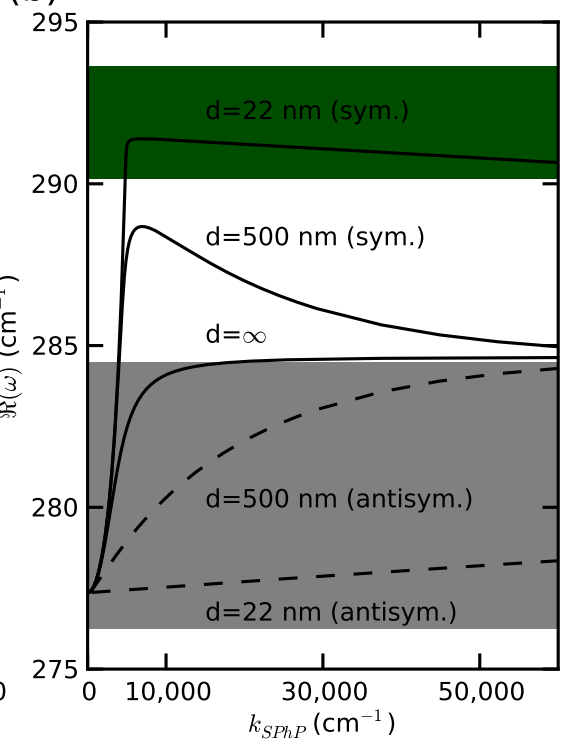

(c)

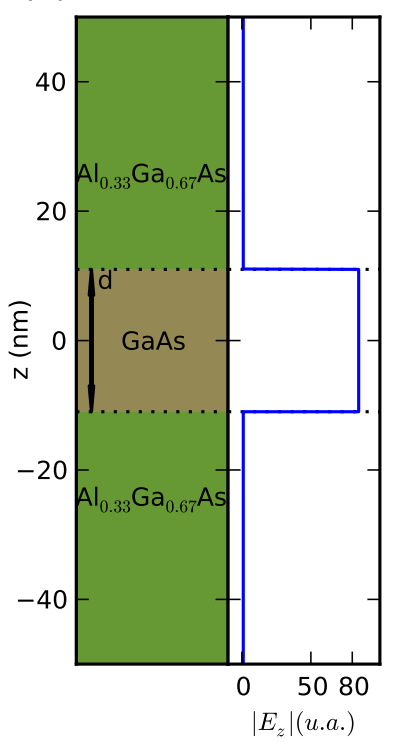

FIG. 1: (Color online) (a) Real part of GaAs and $\mathrm{Al}_{0.33} \mathrm{Ga}_{0.67} \mathrm{As}$ bulk dielectric functions as a function of the frequency. The gray area corresponds to the frequency range where an $\mathrm{SPhP}$ at the $\mathrm{GaAs} / \mathrm{Al}_{0.33} \mathrm{Ga}_{0.67} \mathrm{As}$ single interface can exist. The green (dark gray) shaded area correspond to the ENZ regime of GaAs, defined by $K_{E N Z}=\left|\epsilon_{z z A l G a A s} / \epsilon_{z z G a A s}\right|^{2} \geq 50$. (b) Dispersion relation for the slab geometry and different GaAs thicknesses d. The grey and green (dark gray) shaded areas have the same meaning as in Fig. 1a. When $d=\infty$, the system is similar to a single interface geometry. As the thickness is decreased, the mode separates into two branches (symmetric and antisymmetric normal electric field distribution [22, 23]). When d=22nm, the symmetric branch frequency enters the ENZ regime of GaAs. (c) left: $\mathrm{Al}_{0.33} \mathrm{Ga}_{0.67} \mathrm{As} / \mathrm{GaAs} / \mathrm{Al}_{0.33} \mathrm{Ga}_{0.67} \mathrm{As}$ slab geometry, right: spatial distribution of the z-electric field component of the ENZ mode at $34.3 \mu \mathrm{m}$ for a wavevector of $7000 \mathrm{~cm}^{-1}$ and a GaAs thickness d of $22 \mathrm{~nm}$.

electrical control of THz reflectivity. Other THz modulation schemes have already been demonstrated $[24-$ 27], but they encounter certain difficulties when working at higher frequency $[28]$ and do not work in the optical phonon frequency range of their constitutive materials. Using optical phonons for optoelectronic devices can be surprising as they are usually considered to be detrimental. Indeed, the electron/optical-phonon interaction is a relaxation process much faster than the electron/photon interaction at work in most optoelectronics devices. Here, we use a different scheme based on the resonant absorption by the ENZ mode. Electrons are not used to absorb light but to modulate the ENZ effect. The principle is summarized here: incident light is coupled through a grating to the ENZ mode leading to a resonant absorption. The GaAs layer thickness is adjusted to $22 \mathrm{~nm}$ to form a QW where one intersubband transition (ISBT) energy is close to that of the ENZ mode. Thanks to the ISBT, the z-component of the QW dielectric tensor (see [21] for details) depends on the electron density in the well and can be tuned by a gate voltage. This gives an electrical control of the ENZ enhancement and thus of the absorption, as a lower ENZ enhancement leads to a smaller absorption.

The device is depicted in Fig. 2a. The incident THz field is coupled to the ENZ mode by a grating made of gold stripes which also serves as a Schottky gate for electrical control of the electron density in the single $22 \mathrm{~nm}$ thick GaAs QW. The substrate is a doped GaAs wafer, which behaves as a back-mirror at $\mathrm{THz}$ frequencies. The grating features and spacer thickness were chosen to optimize the absorption in the depleted QW through numerical simulations[21]. Details about the fabrication can be found in [21]. Reflectivity spectra[21], with a p-polarized incident wave (Fig. 2b), show that a $76 \%$ absorption is achieved at $34.3 \mu \mathrm{m}$ (corresponding to the longitudinal optical phonon frequency $8.74 \mathrm{THz}$ ) when a negative gate voltage is applied. This bias depletes the $\mathrm{QW}$, so that the ISBT contribution to the dielectric constant vanishes. No absorption is observed for s-polarization[21]. Numerical simulations, using bulk dielectric constants agree well with the reflectivity data (Fig. 2b). Moreover, they show that $45 \%$ of the incident energy is absorbed within the QW by the ENZ mode. The remaining $31 \%$ is absorbed in the rest of the structure. The calculated near field distribution at resonance confirms the strong $\left|E_{z}\right|^{2}$ enhancement confined in the QW (Fig. 3). Fig. 2c shows that the resonant absorption does not depend on the angle, as expected from the almost flat dispersion relation of the ENZ mode. It is worth comparing this $45 \%$ absorption by the ENZ mode in a single QW with the intersubband absorption of a Quantum Well Infrared Photode- 


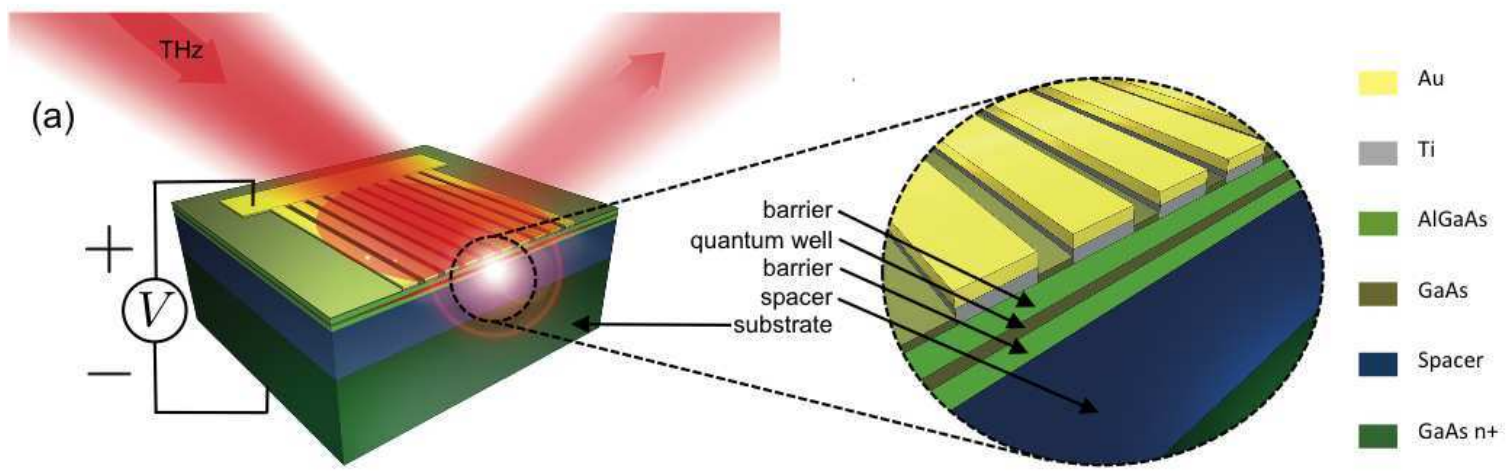

(b)

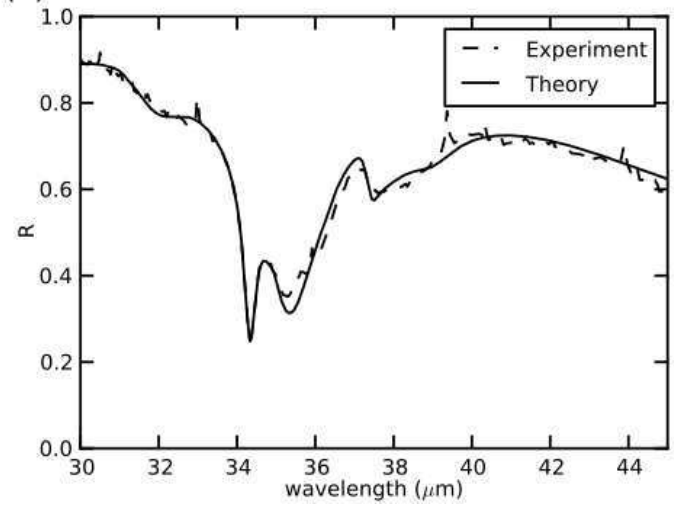

(c)

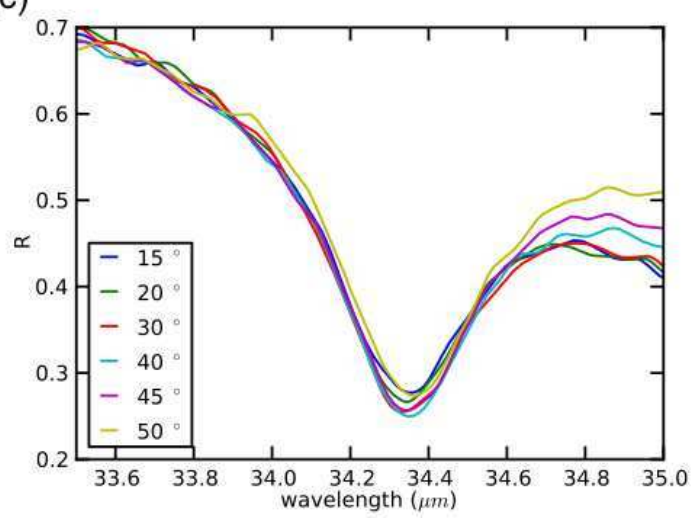

FIG. 2: Excitation of ENZ mode. (a) Scheme of the device made of a gold lamellar grating with $4 \mu \mathrm{m}$ period, $200 \mathrm{~nm}$ thickness, a 0.66 filling factor, a $22 \mathrm{~nm}$ GaAs quantum well buried in $\mathrm{Al}_{0.33} \mathrm{Ga}_{0.67}$ As barriers, a $1300 \mathrm{~nm} \mathrm{Al}_{0.5} \mathrm{Ga}_{0.5} \mathrm{As}$ spacer[21] and a doped GaAs substrate. (b) Reflectivity spectrum for a p-polarized incident wave incident at $15^{\circ}$, with an applied gate voltage of $\mathrm{V}=-0.3 \mathrm{~V}$, data and theory. An absorption peak appears at $34.31 \mu \mathrm{m}$. The incident wave is p-polarized. Note that the second absorption peak near $36 \mu \mathrm{m}$ is due to a surface plasmon propagating on the top of the metallic grating [21]. (c) Reflectivity for various angles of incidence for an applied voltage of $\mathrm{V}=-0.3 \mathrm{~V}$. The absorption peak is almost independent on the angle of incidence. The reflectivity changes as a function of the angle of incidence above $34.6 \mu \mathrm{m}$ are discussed in [21].

tector (QWIP) consisting of 50 QWs, which is typically less than 25\%[29]. Furthermore, we did calculations on the same structure without a grating, and found a maximum absorption in the QW of $4 \%$, only at the Brewster angle.

We now turn to the modulation of the reflectivity. Experimental reflectivity spectra at room temperature for different gate voltages are shown in Fig. 4a. It is seen that at the resonance $(34.3 \mu \mathrm{m})$ the reflectivity decays from 0.38 to 0.25 when the applied voltage goes from $+0.3 \mathrm{~V}$ to $-0.3 \mathrm{~V}$. The corresponding relative variation is as large as $54 \%$ as displayed in Fig. 4b. For larger voltages, the reflectivity change tends to saturate. The physical mechanism is based on controlling the ENZ effect with ISBT in the QW. As previously pointed out, we have an ISBT at the frequency of the ENZ mode (See [21] for more details). An increase of the electron density, through an increase of the gate voltage $(-0.3$ to $+0.3 \mathrm{~V})$ between the metallic grating and the doped substrate, increases the imaginary part of the intersubband contribution to the dielectric constant at the interface mode frequency. Hence $\epsilon_{z z G a A s}$ is no longer close to zero, result-

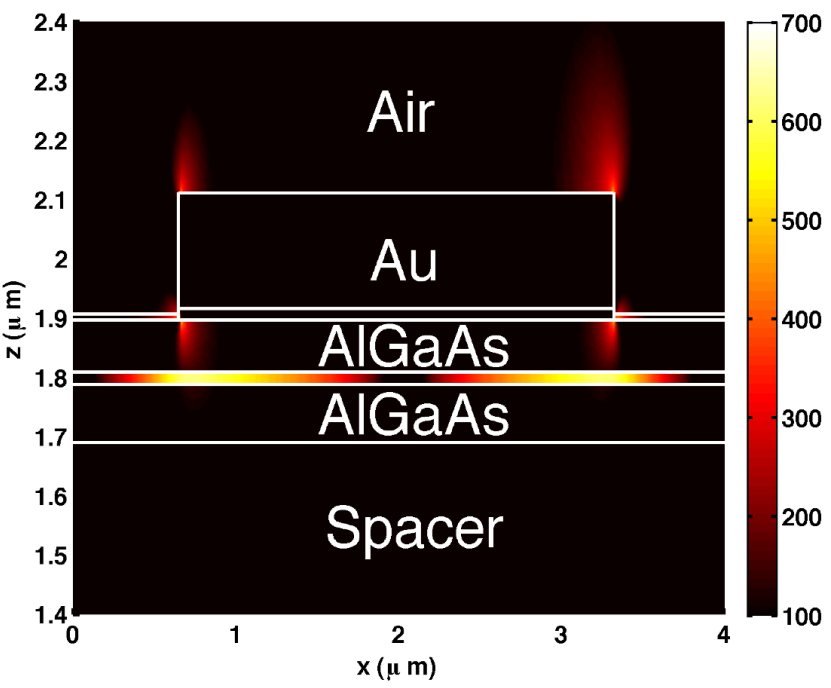

FIG. 3: Normalized near field distribution $\left|E_{z}\right|^{2}$ at the resonance $(34.3 \mu \mathrm{m})$ for a p-polarized plane wave incident at $15^{\circ}$ on the structure. The white lines represent the geometry. Only one period of the grating is represented 
(a)

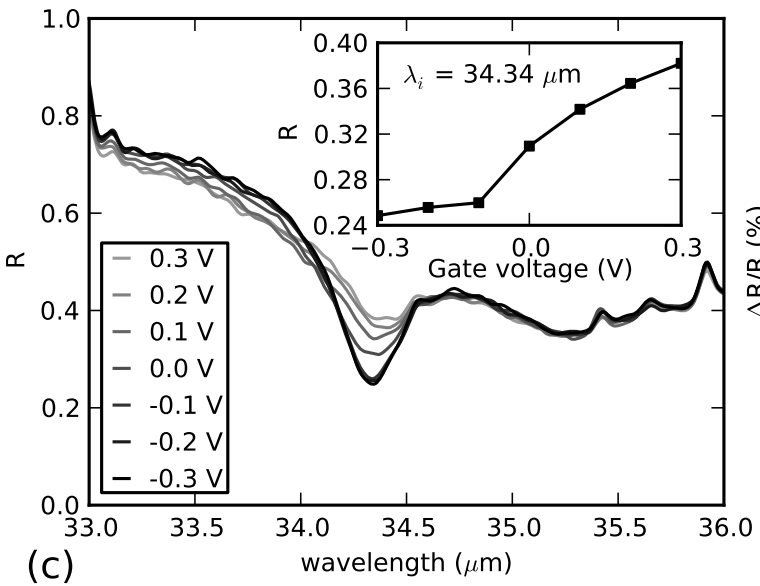

(b)

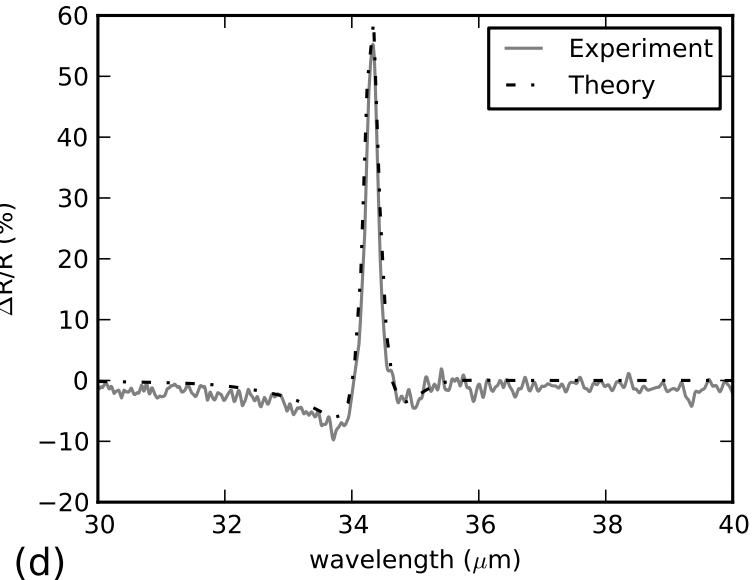

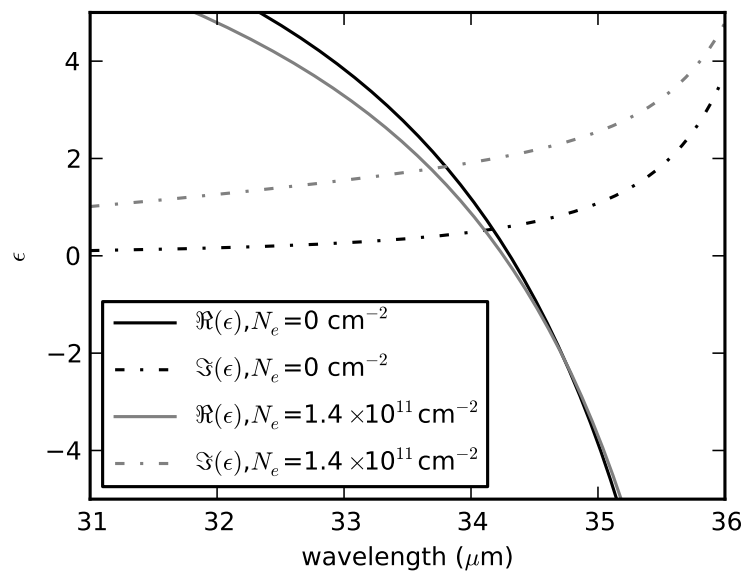

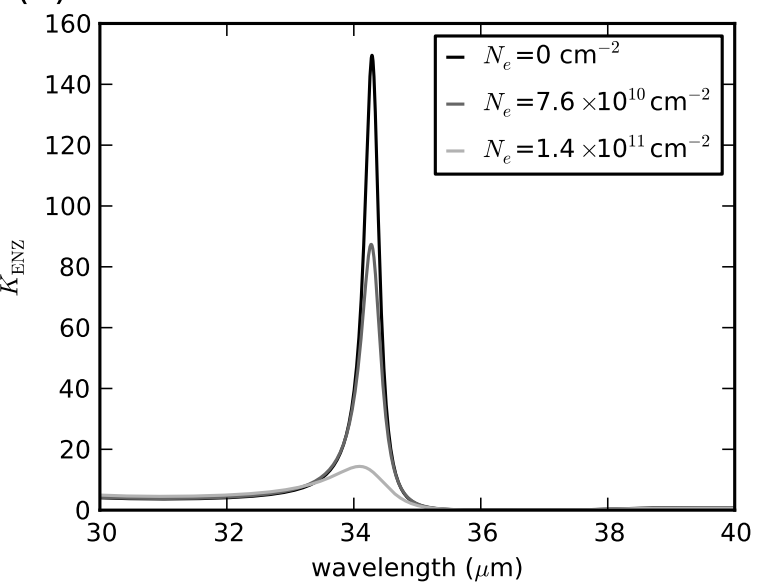

FIG. 4: Electrical modulation of the reflectivity. (a) TM reflectivity for different applied voltages for an angle of incidence of $15^{\circ}$. The inset shows the evolution of the reflectivity at $34.31 \mu \mathrm{m}$ as a function of the gate voltage. (b) Corresponding relative reflectivity modulation between -0.3 and $+0.3 \mathrm{~V}$, data and theory. (c) Dielectric function of GaAs without (electronic density $\left.\mathrm{Ne}=0 \mathrm{~cm}^{-2}\right)$ and with ISBT $\left(\mathrm{Ne}=1.4 \times 10^{11} \mathrm{~cm}^{-2}, \gamma_{i s b}=330 \mathrm{fs}\right)$ the ENZ enhancement factor $K_{\mathrm{ENZ}}$ as a function of the electronic density in the QW.

ing in a suppression of the ENZ field enhancement. By fitting the experimental reflectivity spectrum we obtain the values for the dielectric function for various electronic densities[21] (Fig. 4c) and calculate the corresponding $K_{\mathrm{ENZ}}$ factor that strongly decreases as the electronic density in the QW increases (Fig. 4d). For practical applications, the time response of the device is an important figure of merit. We estimate [21] the maximum bandwidth, mainly limited by the capacitance of the device, to be on the order of $1 \mathrm{GHz}$. Note that the maximum electrical power flowing through the device is $425 \mathrm{nW}$ at $+0.3 \mathrm{~V}$ so that thermal effects are negligible[21].

To summarize, we have introduced an ENZ mode that exhibits strong field enhancement. It can be used to engineer large absorption in a layer as thin as $22 \mathrm{~nm}$ $\left(\approx \lambda_{i} / 1560\right)$. The strong interaction between the ENZ mode and the bidimensional electron gas allows controlling electrically the absorption. A relative change in the reflectivity up to $54 \%$ is experimentally obtained at $8.74 \mathrm{THz}$ and room temperature with a single AlGaAs/GaAs/AlGaAs QW. The ENZ effect is limited to a narrow range of wavelengths for a given material system, but is present in all polar crystals [30]. As a result this effect can be used at various wavelengths such as 11 $\mu \mathrm{m}$ in $\mathrm{SiC}$, or $13.5 \mu \mathrm{m}$ with $\mathrm{GaN}$, where existing modulation scheme become challenging. The control of the ENZ effect appears to be a very promising mechanism to enhance light-matter interaction at the nanoscale. Due to Kirchhoffs law, enhancing and controlling the absorption means that emission can also be enhanced and controlled. Enhancing the ENZ mode electron interaction suggests that electrical transport measurements in the QW should allow the detection of incident radiation. Hence, using the ENZ mode opens new avenues for the design of modulators, sources and detectors. 
[1] W. L. Barnes, A. Dereux, T. W. Ebbesen, Nature 424, 824 (2003).

[2] J.A. Schuller, E.S. Barnard, W. Cai, Y. Chul Jun, J.s. White, M.l. Brongersma, Nature Materials 9, 193 (2010)

[3] S.M. Nie, S.R. Emery, Science 275, 1102 (1997)

[4] ] P. Anger, P. Bharadwaj, L. Novotny, Physical Review Letters 96, 113002 (2006)

[5] S. Kuhn, U. Hakanson, L. Rogobete, V. Sandoghdar, Physical Review Letters 97, 017402 (2006)

[6] K. Drexhage, H. Kuhn, F.P. Schfer, Berichte der Bunsengesellschaft fr Physikalische Chemie 72, 329 (1968)

[7] K. Drexhage, M. Fleck, H. Kuhn, F.P. Schfer, W. Sperling, Berichte der Bunsengesellschaft fr Physikalische Chemie 70, 1179 (1966)

[8] ] R.R. Chance, A. Prock, R. Silbey, Journal of Chemical Physics 60, 2744 (1974)

[9] W.A. Challener, C. Peng, A.V. Itagi, D. Karns, W. Peng, Y. Peng, X. Yang, X. Zhu, N.J. Gokenmeijer, Y.-T. Hsia, G. Ju, R.E. Rottmayer, M.A. Seigler, E.C. Gage, Nature Photonics 3, 220 (2009)

[10] K. Hassan, J.-C. Weeber, L. Markey, A. Dereux, A. Pitilakis, O. Tsilipakos,E. E. Kriezis, Appl. Phys. Lett. 99, $241110(2011)$

[11] M.A. Noginov, G. Zhu, A.M. Belgrave, R. Bakker, V.M. Shalaev, E.E. Narimanov, S. Stout, E. Herz, T. Suteenwong and U. Wiesner, Nature 460, 1110 (2009)

[12] R. F. Oulton, V.J. Sorger, T. Zentgraf, R-M. Ma, C. Gladden, L. Dai, G. Bartal, X. Zhang, Nature 461, 629 (2009)

[13] I. De Leon, P. Berini, Nature Photonics 4, 382 (2010)

[14] JJ.Greffet, R. Carminati, K. Joulain, J-P. Mulet, S. Mainguy, Y. Chen, Nature 416, 61 (2002).
[15] F. Marquier, K. Joulain, J-P. Mulet, R. Carminati, J.J. Greffet, Y. Chen, Phys. Rev. B 69, 155412 (2004).

[16] S. Vassant, F. Marquier, JJ. Greffet, F. Pardo, J-L. Pelouard, Appl. Phy. Lett. 97, 161101 (2010).

[17] S. Enoch, G. Tayeb, P. Sabouroux, N. Guerin, P. Vincent, Phys. Rev. Lett. 89, 213902 (2002).

[18] M. Silveirinha, N. Engheta, Phys. Rev. Lett. 97, 157403 (2006).

[19] B. Edwards, A. Alu, M.E. Young, M. Silveirinha, N. Engheta, Phys. Rev. Lett. 100, 033903 (2008).

[20] N.M. Litchinitser, A.I. Maimistov, I.R. Gabitov, R.Z. Sagdeev, V.M. Shalaev, Opt. Lett. 33, 2350 (2008).

[21] See Supplementary Materials for further explanations.

[22] D. Sarid, Phys. Rev. Lett. 47, 1927 (1981).

[23] P. Berini, Advances in Optics and Photonics 1, 484 (2009).

[24] R. Kersting, G. Strasser, K. Unterrainer, Electron. Lett. 36, 1156 (2000).

[25] T. Kleine-Ostmann, P. Dawson, K. Pierz, G. Hein, M. Koch, Appl. Phys. Lett. 84, 3555 (2004).

[26] H. Chen, W.J. Padilla, J.M.O. Zide, A.C. Gossard, A.J. Taylor, R.D. Averitt, Nature 444, 597 (2006).

[27] W. Maineult, L. Ding, P. Gellie, P. Filloux, C. Sirtori, S. Barbieri, T. Akalin, J-F. Lampin, I. Sagnes, H.E. Beere, D.A. Ritchie, Appl. Phys. Lett. 96, 21108 (2010).

[28] A. Gabbay, J. Reno, J.R. Wendt, A. Gin, M.C. Wanke, M.B. Sinclair, E., Shaner, I. Brener, Appl. Phys. Lett. 98, 203103 (2011)

[29] B.F. Levine, J. Appl. Phys. 74, R1 (1993).

[30] All crystalline materials with more than two different atoms per unit cell are polar and have at least one infrared active optical phonons. In particular, compounds and alloy made of I-VII, II-VI, III-V and IV-IV materials are polar. 